\title{
Role of Interventional Radiology in the Management of Postoperative Biliary Complications \\ ${ }^{1}$ Hana Hamdy Nasif, ${ }^{1}$ Mennatallah Hatem Shalaby, ${ }^{2}$ Mohamed Hosni Kamel Abdelmaksoud, ${ }^{1}$ Ahmed Salama Hussein Mohamed Elsakka \\ ${ }^{1}$ Departemnt of Radiology, Faculty of Medicine, Ain Shams University \\ ${ }^{2}$ Department of Interventional Radiology, Theodore Bilharz research institute, Visiting Scholar, Interventional Radiology, Stanford University, CA, USA
}

Corresponding author: Ahmed S.H.M. Elsakka, Mobile: 01098451004; Email: ahmed.elsakka54@gmail.com

\begin{abstract}
Background: Biliary postoperative complications include biliary stenoses, biliary leaks, fistulas and collection or abscess formation. These complications usually occur due to injury after laparoscopic cholecystectomy, gastric or hepatic resection, bilio-enteric anastomosis and after liver transplantation. In most of the cases a new surgical intervention is not possible. Endoscopic intervention is usually the optimal initial management of these complications; however, in patients with altered enteric passage, tight low biliary stricture, high up obstruction or leakage endoscopic approach becomes impossible. Therefore, the percutaneous trans-hepatic approach is of extreme importance in the diagnosis and treatment of the problem especially in complex cases Objective: This study aimed to highlight the spectrum of percutaneous cholangiographic findings and methods of treatment of postoperative benign biliary stenoses, biliary leaks and to detect its significance in management of these problems.

Patients and Methods: This study was conducted in Theodor bilharz research institute (TBRI), Egypt from February 2013 to June 2018. 17 patients with benign postoperative biliary complications (stricture and leak) were treated with a percutaneous transhepatic procedure. Only adult patients (age $>18$ years) were included in the study.

Results: All patients had cholestasis and/or bile leak according to clinical and biologic findings (i.e., jaundice or an episode of cholangitis). Biliary obstruction and/or leak was confirmed by abdominal ultrasonography (US), multidetector computed tomography, and/or magnetic resonance (MR) cholangiography when US results were not conclusive and confirmation of the site \& extension of the stricture and/or leak was needed. The results of the present study demonstrated that treatment of post-operative biliary complications (stricture and/or leak) by percutaneous transhepatic procedures was an effective option with acceptable rate of complication.

Conclusion: We concluded that the percutaneous treatment of post- operative biliary complications is an effective alternative to surgery. This method should certainly be preferred when the local conditions were not favorable to a surgical repair, failure of endoscopic approach or when a recurrence was observed after an unsuccessful previous repair. Its advantages compared with surgery were its minimally invasive nature, reduced risk of complications, and the fact that all options remain open in case of failure.
\end{abstract}

Keywords: Ultrasonography - Magnetic resonance - Percutaneous transhepatic biliary drainage

\section{INTRODUCTION}

Bile duct injuries are an infrequent but potentially devastating complication of biliary tract surgery, with cholecystectomy accounting for the largest proportion of such injuries. The annual incidence of bile duct injuries increased from approximately $0.2 \%$ in the era of open cholecystectomy to approximately $0.5 \%$ after laparoscopic cholecystectomy became widely available. Biliary injuries are associated with high morbidity and mortality, impaired quality of life, and substantial financial burdens to patients and society ${ }^{(1)}$.

Optimal management of biliary injuries is achieved with a multidisciplinary approach. Successful management depends on the type of injury, timing of injury recognition, presence of complications, condition of the patient, and availability of experienced hepatobiliary surgeons. Radiologists play a key role in diagnosis and treatment. Imaging is vital for initial diagnosis, assessment of the extent of injury, and preprocedural planning. Depending on the type of injury, appropriate management methods may include endoscopic, percutaneous, and surgical interventions ${ }^{(l)}$.

Interventional radiology (IR) has an established role in the diagnosis and management of patients with post-operative biliary complications. Postoperative benign biliary stenoses and/or biliary leaks and fistulas may be effectively treated with dilatation of the narrowed anastomotic tract or bile diversion away from the site of defect in the bile wall. Drain collection and tract embolization are the other options for bile leak percutaneous treatment. A large variety of 
combination of dilatation and drainage may be used on a "case-by-case" basis with the use of various techniques and materials ${ }^{(2)}$.

\section{AIM OF THE WORK}

This study aimed to highlight the spectrum of percutaneous cholangiographic findings and methods of treatment of postoperative benign biliary stenoses, biliary leaks and detect its significance in management of these problems.

\section{PATIENTS AND METHODS}

This study was conducted in Theodor bilharz research institute (TBRI), Egypt from February 2013 to June 2018.

17 patients with benign postoperative biliary complications (stricture and leak) were treated with a percutaneous transhepatic procedure. These patients constituted the cohort of this singlesite retro-spective study.

The local ethical committee of TBRI Hospital approved the study protocol. In addition, a written informed consent was obtained from all patients' prior recruitment to study.

Only adult patients (age >18 years) were included in the study. All patients had cholestasis and/or bile leak according to clinical and biologic findings (i.e., jaundice or an episode of cholangitis). Biliary obstruction and/or leak was confirmed by abdominal ultrasonography (US), multidetector computed tomography, and/or magnetic resonance (MR) cholangiography when US results were not conclusive and confirmation of the site \& extension of the stricture and/or leak was needed.

The level and extension of biliary duct stenosis, the presence of gall stones, and other pathologic processes were assessed on imaging to plan a management strategy. All biliary stenoses and/or leak cases were then confirmed by direct percutaneous cholangiography.

Patients with a history of malignant biliary disease, associated vascular injuries during the operation and associated contraindications to the procedure such as massive ascites or post endoscopic pancreatitis were excluded from the study. In addition, pregnant females and patients had Contraindications to general anesthesia were excluded from the study.
Our treatment strategy used in treatment of different biliary complications was:

In case of biliary stricture : Repeated balloon dilatation every 2-3 weeks through the internal-external drainage catheter with oversizing balloon from 6 to 12 $\mathrm{mm}$ in diameter until good cholangiographic results are obtained and clinical condition of the patient and laboratory investigations were improved .8 to 10 French plastic stent could be used if cholangiographic results after repeated balloon dilatation are not satisfactory.

In case of biliary leakage : Internal-External drainage catheter was done to divert the bile away from the site of defect in the bile wall, to drain thoroughly any collections and to seal the tract if possible and catheter is replaced every 2 to 3 months. Cholangiogram was obtained every 2-3 months until healing of the site of the leakage was achieved. According to the last cholangiographic image, 8 to 10 French plastic stent could be used if leakage was still present even after repeated exchange of the InternalExternal drainage catheter.

In case of biliary stricture associated with leakage : Internal-External drainage catheter was inserted and replaced every 2 to 3 months until healing of the site of the leakage was achieved. According to the last cholangiographic picture, balloon dilatation might or might not be used.

\section{RESULTS}

A total of 17 patients (13 women and 4 men; age, 28- 76 years; mean age, $40 \mathrm{y}$ ) were referred for percutaneous transhepatic procedures between February 2013 and June 2018 (Table 1).

Table (1): Patient Characteristics.



Figure (1): Diagram shows Causes of post-operative biliary complications. 
The underlying causes of biliary complications included biliary injury during laparoscopic cholecystectomy in 8 patients, hepaticojujenostomy in 4 patients, choledochojejunostomy in 2 patients, laparoscopic cholecystectomy converted to open in 1 patient, open cholecystectomy in 1 patient and pancreaticodudenectomy (Whipple operation) in 1 patient ( Table 2) (Figure 1).

Table (2): Causes of post-operative biliary complications.

\begin{tabular}{|l|l|}
\hline Initial Surgery & $\begin{array}{l}\text { No. of } \\
\text { Cases }\end{array}$ \\
\hline Lap. Cholecystectomy & 8 \\
Lap. Cholecystectomy \& Hepaticojejunostomy & 4 \\
Lap. Cholecystectomy \& Choledochojejunostomy & 2 \\
Lap. Cholecystectomy converted to open & 1 \\
Open cholecystectomy & 1 \\
Pancreaticodudenectomy (Whipple operation) & 1 \\
\hline
\end{tabular}

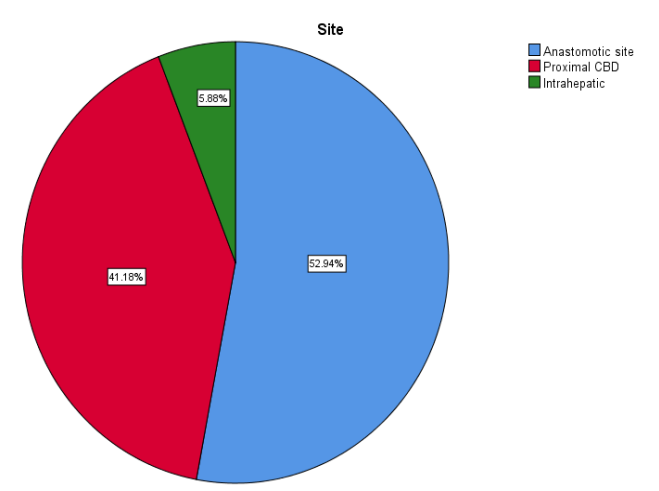

Figure (2): Diagram shows different locations of stricture and/or leak.

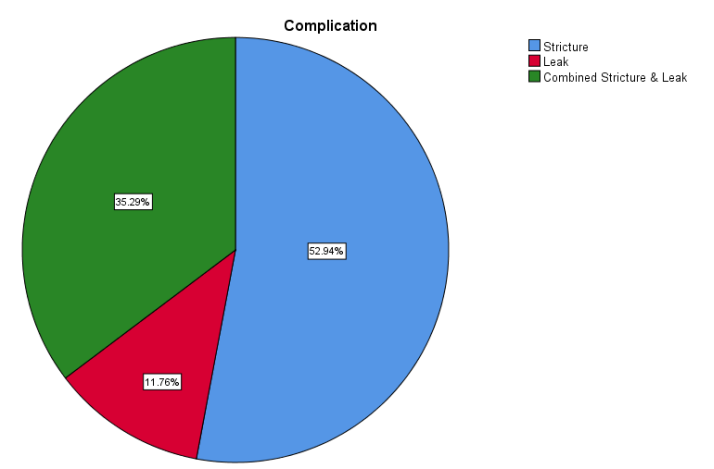

Figure (3): Diagram shows percentage of different types of biliary complications.

The exact site of biliary stricture and/or leak was anastomotic in 9 patients, Proximal CBD in 7 patients and intrahepatic in 1 patient (Figure 2).

Biliary complications included stricture in 9 patients, leak in 2 patients and combined stricture \& leak in 6 patients (Figure 3).
The percutaneous procedure time ranged between 40 to 60 minutes but it consumed more time in difficult cases such as tight stenosis or challenging biliary access in non-dilated systems.

Thirty four internal/external catheters were inserted in 17 patients (mean 2-3 catheters for every patient).

Right side transhepatic approach done in 14 patients. Left sided approach done in 3 patients.

Percutaneous biliary drainage without cholangioplasty was done in 3 patients. PTBD and balloon angioplasty was performed in 8 patients with use of angioplasty balloon catheters placed across the strictures. The caliber of the balloon ranged from 6 $\mathrm{mm}$ to $12 \mathrm{~mm}$. 6 Patients who failed PTBD plus balloon cholangioplasty underwent plastic stent insertion.

The mean number of balloon dilation sessions was three. The tracts were dilated in a stepwise fashion with dilators (size range, $6-12 \mathrm{~F}$ ) to accept an internal/external catheter.

In the stent group (6 patients), 8 to $10 \mathrm{~F}$ plastic stent inserted.

Percutaneous plastic stent insertion done in 3 patients. The Rendezvous Technique used to insert plastic stent in the other 3 patients. This technique is a fluoroscopic and endoscopic maneuver in which there is a "meeting" at the papilla between a wire guide that enters through a percutaneous tract and the duodenoscope that it is placed in front the papilla. During this encounter, when the wire guide exits through the papilla, it is caught with a snare and is withdrawn through the accessory channel of the duodenoscope.

The duration of drainage ranged from 120 to 365 days (mean, $150 \mathrm{~d}$ ).

Definite leakage control was achieved in all patients who had biliary leak after 2 to 3 months of PTBD except on patient who received plastic stent by rendezvous technique after 1 month of biliary drainage.

15 patients out of 17 had completed the treatment protocol. Two patients still have internalexternal biliary drainage catheter.

The mean duration of follow up after completion of treatment strategy ranged between 6 months to 1.5 years (mean 7 months). 


\section{Laboratory Parameters}

Laboratory parameters included liver function tests (ALT, AST, Bilirubin and ALP), HB level, WBCS count and C-reactive protein (inflammatory marker).

All these parameters were measured at base level (pre-intervention) and after completion of treatment protocol.

Mean pre-intervention bilirubin level was 5.8 $\mathrm{mg} / \mathrm{dl} \pm 4.9$ compared to $1.98 \mathrm{mg} / \mathrm{dl} \pm 1.95$ postintervention with $\mathrm{P}$-value of 0.002 (statistically significant) and 95\% CI (1.6-5.9).

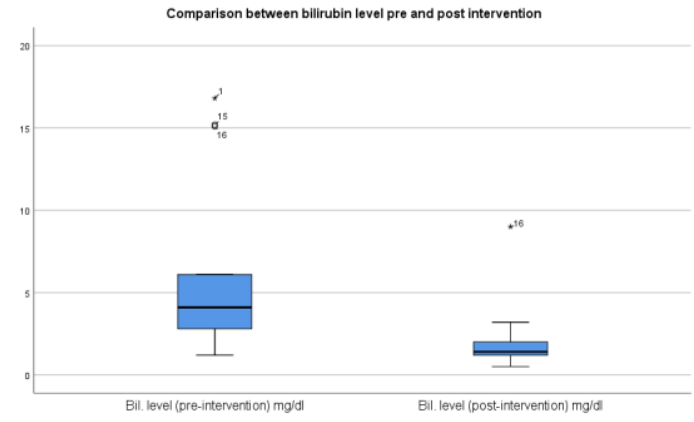

Mean pre-intervention liver enzymes (ALT and AST levels) were consecutively 92.3, 94.2 U/L \pm 67.2, 11.6 compared to 37, $41 \mathrm{U} / \mathrm{L} / \mathrm{dl} \pm 11.6,18.7$ post-intervention with P-value of 0.002 (statistically significant) and 95\% CI (24-86.6), (23.8 -82.3).
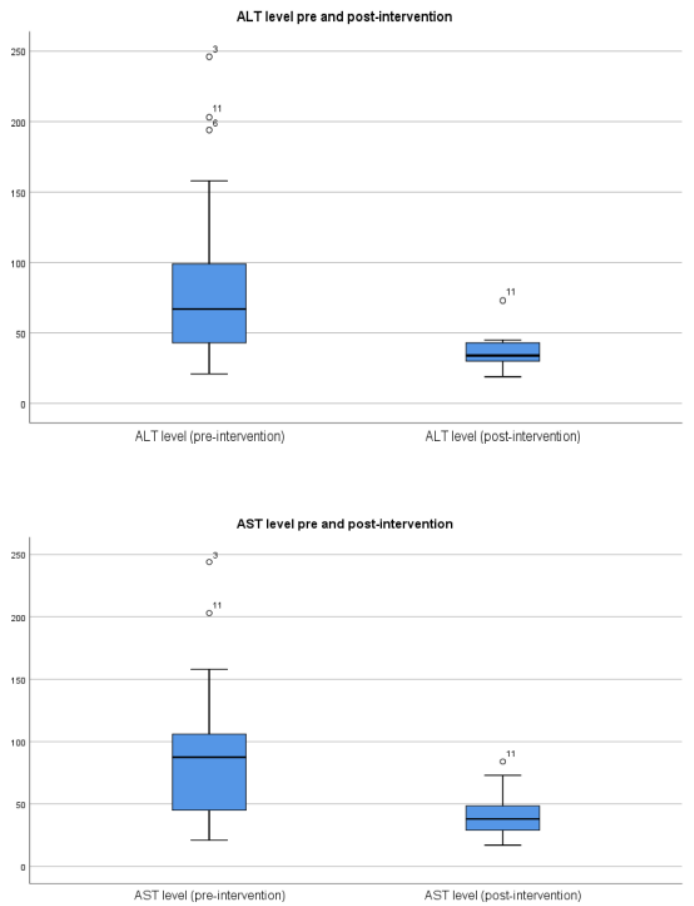

Mean pre-intervention alkaline phosphatase (ALP) level was 566.9 U/L \pm 485.2 compared to $108.8 \mathrm{mg} / \mathrm{dl} \pm 53.7$ post-intervention with P-value of 0.001 (statistically significant) and 95\% CI (226.9689.2).

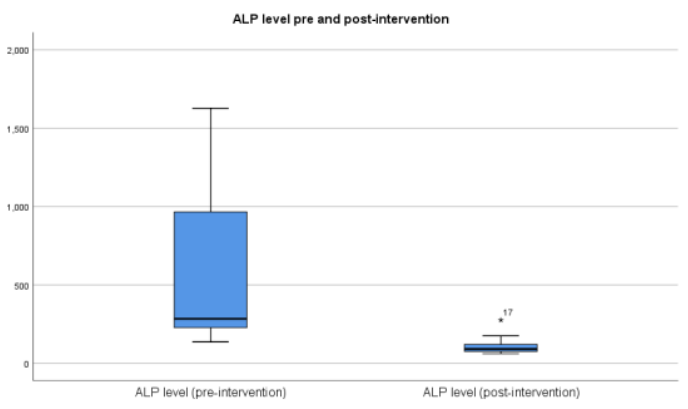

Mean pre-intervention CRP level was 151 $\mathrm{mg} / \mathrm{l} \pm 80.8$ compared to $30.8 \mathrm{mg} / \mathrm{l} \pm 17.7$ postintervention with $\mathrm{P}$-value of 0.009 (statistically significant) and 95\% CI (43.3-196.9).

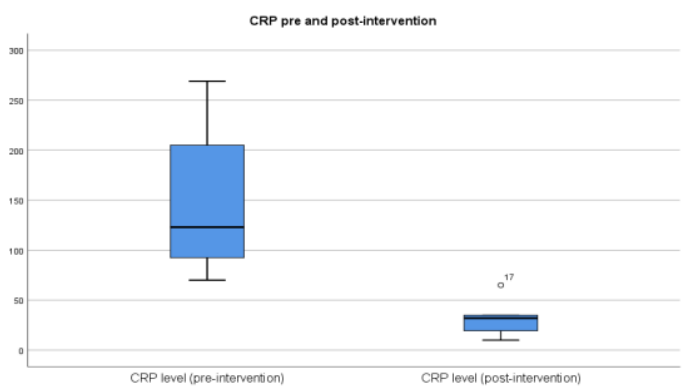

Mean pre-intervention WBCS count was 11.4 thousands $/ \mathrm{cmm} \pm 6.1$ compared to 7.1 thousands $/ \mathrm{cmm}$ \pm 2.3 post-intervention with P-value of 0.004 (statistically significant) and 95\% CI (1.5-6.9).

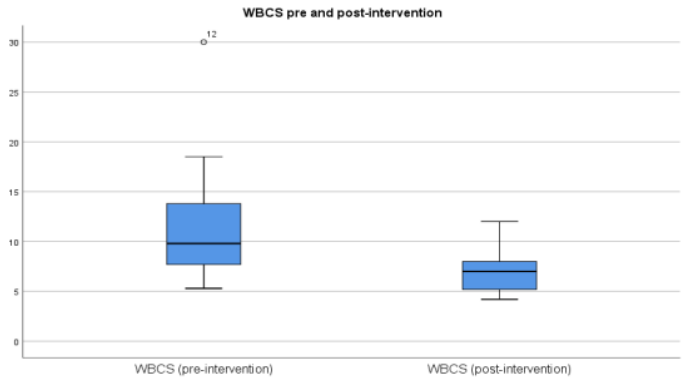

Mean pre-intervention hemoglobin level was $10.2 \mathrm{gm} / \mathrm{dl} \pm 2$ compared to $11.3 \mathrm{gm} / \mathrm{dl} \pm 1.5$ postintervention with $\mathrm{P}$-value of 0.002 (statistically significant) and 95\% CI (-1.7, -0.47). 


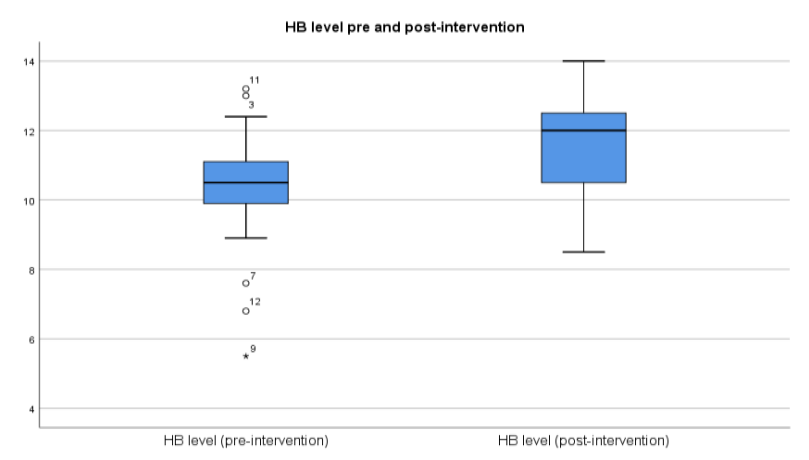

\section{Complications}

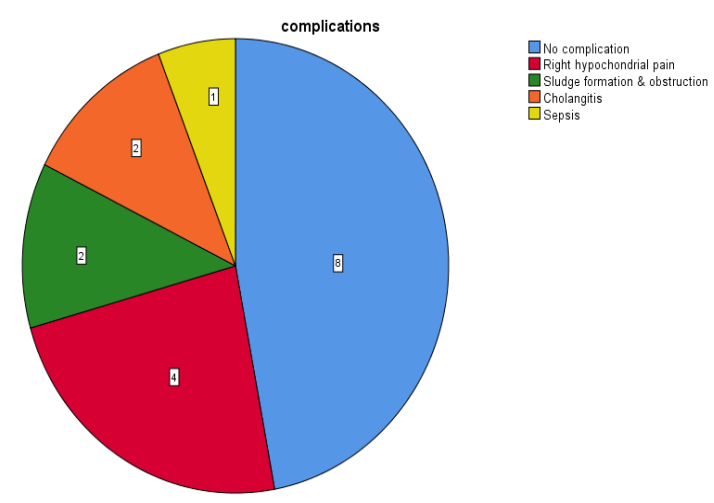

Figure (4): Diagram shows frequency of different procedural complications.

Three patients had major complications: two patients had cholangitis and one patient had sepsis. Minor complications in 6 patients included catheter dislodgment or obstruction (2 patients), and right hypochondrial pain (4 patients). These minor complications were managed on an outpatient basis. 8 patients had no complication (Figure 4).

\section{DISCUSSION}

The results of the present study demonstrated that treatment of post-operative biliary complications (stricture and/or leak) by percutaneous transhepatic procedures was an effective option with acceptable rate of complication.

In cases of bile duct stricture, surgical reconstruction was considered the treatment of choice, with long-term success rates ranging from $80 \%$ to $90 \%$. However, surgical reconstruction was associated with mortality rates as high as $2.2 \%$ and morbidity rates as high as $33 \%$. In addition, recurrence of the stricture was the main complication of surgical repair, occurring in as many as $25 \%$ of patients within a 5 -year follow-up $\operatorname{period}^{(3)}$.
Several factors have been associated with an increased risk of failure, such as age, chronic liver disease, level of stricture and involvement of inexperienced hepatobiliary surgeons ${ }^{(3)}$.

Comparison between long-term results of surgery and interventional techniques has been made. Pitt et al. ${ }^{(4)}$ reported superior results for surgery ( $88 \%$ success rate vs $55 \%$ ) with a mean follow-up of 59 months. They compared surgical repair and percutaneous balloon dilation in 45 patients with a standardized management protocol in a nonrandomized population; the choice of management technique was based on patient preference. Moreover, surgical repair included pre- and postoperative percutaneous biliary drainage in place for a mean duration of 13.8 months after surgery. In a retrospective study of 101 patients with benign biliary stricture (i.e. surgery or traumatic) treated by surgery $(n=35)$ and endoscopic stent implantation $(\mathrm{n}=66)$, Davids et al. ${ }^{(5)}$ demonstrated similar long-term success rates.

Currently, endoscopic management of benign biliary stricture is well established and is performed by balloon dilatation and insertion of one or more plastic stents to calibrate the stricture. Many studies reported long-term success rates as high as $83 \%$ for benign biliary strictures with low mortality rates. The main limitation of the endoscopic approach includes the inability to cannulate a bilioenteric anastomosis ${ }^{(3)(6)(7)}$.

The use of percutaneous techniques to treat biliary strictures was first reported in 1974. A multicenter review reported that percutaneous transhepatic drainage provided a $67 \%$ patency rate for anastomotic strictures after 3 years of follow-up (3).

Williams and Bender (6) demonstrated successful dilation in $73 \%$ of patients with bilioenteric anastomosis stricture, with a mean duration of 28 months of follow- up.

Recently, House et al. ${ }^{(7)}$ demonstrated that $90 \%$ of patients with benign bile duct stricture could be treated successfully with a percutaneous biliary approach (i.e., catheter, dilation, or stent placement).

Ludivine et al. ${ }^{(3)}$ demonstrated that patency rate of $70.6 \%$ in bilio-enteric anastomotic strictures during a mean duration of follow up of 34 months compared to 18 months in our study. Their protocol 
was to repair the stricture segment by calibration of the stenosis to $15 \mathrm{~F}$ with a silicone drain and perform internal/external drainage for at least 1 year compared to our protocol which depended on repeated balloon dilatation every 2-3 weeks with exchange of the internal/external drainage catheter every 2-3 months. Their major complication rate was $7 \%$ compared to $17 \%$ in our study.

Treatment of benign bile duct strictures with uncovered metallic stents via an endoscopic or percutaneous approach had been proposed in the past 10 years. Initial studies identified excellent results in the treatment of benign bile duct strictures, with $82 \%$ patency rates after 8 months of follow-up ${ }^{(3)}$.

Nevertheless, series have demonstrated high rates of recurrent occlusion in long-term follow-up. Uncovered metallic stent treatment was generally not accepted in benign biliary disease because it compromised surgical options and had short-term patency ${ }^{(3)}$.

Retrievable fully covered metallic stents could be very good alternative in management of benign biliary disease in terms of patency rate and duration of percutaneous transhepatic biliary drainage (PTBD) but have high rate of migration (11-27\% in most studies). Kim et al. ${ }^{(8)}$ compared retrievable fully covered metallic stents versus percutaneous balloon dilation in the treatment of benign biliary strictures. The primary patency rates were significantly better in the stent group $(87 \%$ at 3 years) than in the balloon group (44\% at 3 years; $\mathrm{P}=.022$ ). Also the indwelling period of PTBD catheters after the initial procedure was able to be significantly reduced in the stent group (median 2.5 months) compared with the balloon group (median 4.5 months; $\mathrm{P}=.001$ ) but stent migration occurred in $6 \%$ of the stent group.

Biodegradable stents are recently introduced in the management of benign biliary stricture with very promising results. They showed improved quality of life and no risk of migration. These stents are tailored specifically for each patient, did not need to be removed after implantation, and offered improved patency owing to their lower biofilm accumulation and reduced incidence of bile duct proliferative changes.

Dopazo et al. ${ }^{(9)}$ study demonstrated the role of biodegradable stents as a part of treatment of biliary strictures after pediatric and adult liver transplantation and anastomotic stricture. It showed high rate of success (more than 80\%) after 2 years of follow up with no stent migration during follow up period.

Cholangioplasty remained controversial in terms of technical aspects regarding size and length of balloon, frequency of dilation, pressure level during dilation, and duration of stent maintenance. The maximum size of the balloon is based on the diameter of the proximal bile duct, which is $8-12$ $\mathrm{mm}$, and the duration of balloon maintenance is between 30 seconds and 20 minutes.

In this study, we used 6-12 mm balloon for 1 to 2 minutes every session. The balloon cholangioplasty could be repeated in the same session according to cholangiographic results after first cycle.

Vos et al. ${ }^{(10)}$ reported a $93 \%$ initial success rate and an $80 \%$ success rate at 33 months after percutaneous balloon dilation for benign hepaticojujenostomy strictures. They performed multiple dilations (maximum of three per patient which is comparable to this study; mean of four dilations; maximum balloon diameter, 7-10 mm) during a period of 1-13 weeks. The procedure was repeated until no more residual stricture was identified.

Schumacher et al. ${ }^{(11)}$ used a calibration of the stenosis to $16 \mathrm{~F}$ compared to 8 to $10 \mathrm{~F}$ in our study.

Some recent reports described the use of a cutting balloon to perform bile duct dilation. This emerging technology offered easier dilation and improved long-term patency in the management of bile duct strictures of as much as $90 \%{ }^{(3)}$.

The duration of treatment to avoid recurrence of stricture was controversial. Some authors suggested the use of short-term stent implantation because, theoretically, fibrotic reactions may be induced by long-term drainage. Conversely, some authors claimed that long-term stent implantation allows better stabilization of inflammatory fibrosis at the site of the stricture and therefore minimized the risk of recurrence. Most studies concluded that the results were excellent with large catheters during 12 months of treatment (3).

Williams and Bender ${ }^{(6)}$ suggested that biliary enteric strictures required stent implantation 
for a significantly longer period than primary biliary duct injuries ( 8.8 months vs 4.7 months).

In our procedure, like those of some other authors, the final decision to remove the biliary internal drainage catheter was based on the appearance of the stenotic segment or leakage site on cholangiography associated with the absence of recurrent clinical/biologic symptoms. Some authors used clinical and manometric perfusion tests to decide on ablation of the bile duct catheter.

The clinical test consisted of placement of a safety catheter above the biliary stricture. The catheter was clamped for 1-2 weeks and removed when no signs or symptoms of cholangitis were observed, along with no changes in liver enzymes. If the patient remained asymptomatic during the clinical trial, and if there was a documented evidence of flow across the stricture on follow-up cholangiography, a biliary manometric perfusion test was performed. A biliary pressure less than 20 $\mathrm{cm} \mathrm{H} 2 \mathrm{O}$ was considered normal.

Savader et al. ${ }^{(12)}$ compared manometric perfusion tests with clinical tests and found the same predictive value for long-term patency of benign bile duct strictures treated by dilation and drainage. We did not base removal of the catheter on the results of manometry.

Koops et al. ${ }^{(13)}$ demonstrated successful PTBD-placement in 18 out of 20 patients who had biliary leak after upper abdominal surgeries. Definite leakage control (DLC) was achieved in 10 patients $(55 \%)$ compared to 8 patients in our study $(100 \%)$. Mean duration of PTBD-therapy in patients with definite leakage control was 31 days compared to 30 to 60 days in our study.

Recent quality improvement guidelines for percutaneous transhepatic cholangiography and biliary drainage suggested thresholds of $5 \%$ each for sepsis, hemorrhage, and localized infection, $2 \%$ thresholds for pleural complications; and a death rate of $3 \%{ }^{(3)}$.

In light of the threshold values, our major complication rates were excellent. Major complications occurred in three of 17 patients (cholangitis and sepsis) without mortality. Minor complications occurred in 6 patients and included mild right hypochondrial pain, sludge formation and catheter dislodgement.
Our data demonstrated that percutaneous therapy is an effective and low-risk procedure with acceptable short-term results for the treatment of patients with benign post-operative biliary stricture and/or leak.

Although no clear therapeutic algorithm has been reported, there had been many approaches to treat bile duct stenosis including balloon dilation with or without stent placement or calibration stent implantation using large drainage catheters with prolonged internal drainage.

The main limitations of the present study were that the study design was retrospective and may have led to an underestimation of the rate of minor complications. Also the follow-up in our study was approximately 1.5 years which was shorter than other studies and this leads to inability to calculate the long-term patency rate.

Our results were excellent on short-term, with an acceptable rate of complication. We were unable to prove that percutaneous treatment could successfully prevent late complications such as biliary cirrhosis.

\section{CONCLUSIONS}

In conclusion, the management of post-operative biliary complications required a thoughtful approach by a multidisciplinary team. The appropriate procedure might be chosen according to an individual basis. We concluded that the percutaneous treatment of post-operative biliary complications was an effective alternative to surgery. This method should certainly be preferred when the local conditions are not favorable to a surgical repair, failure of endoscopic approach or when a recurrence was observed after an unsuccessful previous repair. Its advantages compared with surgery were its minimal invasive nature, reduced risk of complications, and the fact that all options remained open in case of failure.

\section{REFERENCES}

1. Colin MT, Nael ES, Robin RQ et al. (2013): Management of Iatrogenic Bile Duct Injuries: Role of the Interventional Radiologist. Radio Graphics, 33(1):117-134.

2. Miltiadis $\mathrm{K}$, Gianluigi $\mathrm{O}$, Michele $\mathrm{R}$ et al. (2013): Interventional radiology in the management of benign biliary stenoses, biliary leaks and fistulas: a pictorial review. Insights Imaging, 4(1):77-84. 
3. Ludivine G, Marion C, Sabine F et al. (2008): Long-term Outcome of Percutaneous Transhepatic Therapy for Benign Bilioenteric Anastomotic Strictures. J Vasc Interv Radiol., 19:1336-1343.

4. Pitt HA, Kaufman SL, Coleman J et al. (1989): Benign postoperative biliary strictures: operate or dilate? Ann Surg., 210:417-425.

5. Davids PH, Tanka AK, Rauws EA et al. (1993): Benign biliary strictures: surgery or endoscopy? Ann Surg., 217:237-243.

6. Williams HJ and Bender CE (1987): Benign postoperative biliary strictures: dilation with fluoroscopic guidance. Radiology, 163:629634.

7. House MG, Cameron JL, Schulick RD et al. (2006): Incidence and outcome of biliary strictures after pancreaticoduodenectomy. Ann Surg., 243:571-576.

8. Kim JH, Dong Il Gwon, Gi-Young Ko et al. (2011): Temporary Placement of Retrievable Fully Covered Metallic Stents versus Percutaneous Balloon Dilation in the Treatment of Benign Biliary Strictures. J Vasc Interv Radiol., 22:893-899.
9. Dopazo C, Iratxe D, Jesús $Q$ and Anna C (2018): Role of Biodegradable Stents as Part of Treatment of Biliary Strictures after Pediatric and Adult Liver Transplantation: An Observational Single-Center Study. J Vas Interv Radiol., 29:899-904.

10. Vos PM, van Beek EJ, Smits NJ et al. (2000): Percutaneous balloon dilatation for benign hepaticojejunostomy strictures. Abdominal Imaging, 25:134-138.

11. Schumacher B, Othman $T$ et al. (2001): Long-term follow- up of percutaneous transhepatictherapy (PTT) in patients with definite benign anastomotic strictures after hepaticojejunostomy. Endoscopy, 33:409-415.

12. Savader SJ, Cameron JL, Pitt HA et al. (1994): Biliary manometry versus clinical trial: value as predictors of success after treatment of biliary tract strictures. J Vasc Interv Radiol., 5:757-763.

13. Koops A, Nentwich MF, Ittrich H, Bockhorn M et al. (2014): Percutaneous transhepatic biliary drainage in non-cholestatic patients as treatment for bile leaks after upper abdominal surgery. J Vasc Interv Radiol., 25: S49. 